\title{
Hipertensión Portal en niños
}

\author{
DR. JOSE ZACARIAS * y DR. PATRICIO BRINCK *.
}

El Sindrome de Hipertensión Portal (H. P.) resulta de la dificultad del flujo venoso en cualquier punto del trayecto que va desde el lecho capilar de la pared intestinal y del bazo, pasando luego por los sinusoides hepáticos hasta liegar a través de la suprahepatica hacia la Vena Cava Inferior. En los adultos la causa más frecuente de Hipertensión Portal es la cirrosis hepática, enfermedad que no ocupa un lugar preponderante entre las etiologías de este sindrome en niños.

Afortunadamente es una patología poco frecuente en niños cuyo pronóstico está condicionado fundamentalmente por dos factores: la edad y etiología de la Hipertensión Portal. Hay acuerdo entre los autores que se han preocupado de este tema en que las anastomosis portosistémicas antes de $\operatorname{los} 6$ años son de malos resultados y que la edad más adecuada sería después de los 8 a 10 años (1). El mal pronóstico en relación con la etiología está condicionada por aquellas Hipertensión Portal secundarias a cirrosis hepática que irreversiblemente en un plazo menor o mayor llevan a la muerte.

Se revisaron las manifestaciones clínicas, hallazgos de laboratorio, evolución, complicaciones y tratamientos indicados en los pacientes que ingresaron al Hospital "Luis Calvo Mackenna" por Hipertensión Portal en un período de 6 años. El objetivo de este trabajo es llegar a obtener conclusiones sobre técnicas de diagnóstico y tratamiento que permitan un mejor manejo de estos enfermos para alcanzar una edad adecuada, logrando así un mejor rendimiento del tratamiento quírúrgico.

Material clinico. Se revisaron las fichas clínicas de 12 pacientes con Hipertensión Portal controlados en el Departamento de Hepatología del Hospital "Luis Calvo Mackenna" en el período 1968-1974. El diagnóstico fue fundamentado en el cuadro clínico y exámenes de laboratorio. Entre estos últimos se dio especial importancia a

\footnotetext{
* Departamento de Pediatria. Hospital Luis Calvo Mackenna.
}

la radiografía de esófago con medio de contraste; esplenoportografía utilizando como medio de contraste Hypaque al $75 \%$ en una cantidad de 12 20 cc. y a la biopsia bepática. Sólo en dos casos se determinó la presión venosa hepática impactada.

Resultados. De los pacientes estudiados, en 8 se diagnosticó Hipertensión Portal por obstrucción de la Vena Porta. En la tabla No 1 aparecen las manifestaciones clínicas más relevantes de estos pacientes. Se observó mayor frecuencia en varones menores de 4 años de edad; sólo en un

TABLA No 1

HIPERTENSION PORTAL

FIBROSIS HEPATICA CONGENITA

\begin{tabular}{lccc}
\hline Número casos & 1 & 2 & $\beta$ \\
\hline Edad (años) & $5-8 / 12$ & 6 & 5 \\
Sexo & M & M & F \\
Antecedentes & As. Crónico & As. Crónico & $(-)$ \\
$\begin{array}{l}\text { Esplenomegalia } \\
\text { (cms.) }\end{array}$ & 5 & 3 & 6 \\
$\begin{array}{l}\text { Hepatomegalia } \\
\text { (cms.) }\end{array}$ & NO & 2 & NO \\
$\begin{array}{l}\text { Plaquetopenia } \\
\text { Leucopenia }\end{array}$ & NO & NO & ++ \\
$\begin{array}{l}\text { Hemorragia } \\
\text { Digestiva }\end{array}$ & + & NO & ++ \\
\hline
\end{tabular}

caso existía el antecedente exanguíneo transfusión en el período de Recién Nacido. Todos presentaban esplenomegalia y sólo dos hepatomegalia.

El Sindrome de Hiperesplenia manifestado con descenso de las plaquetas y leucopenia estuvo presente en mayor o menor intersidad en todos ellos. En la radiografía de esófago con medio de contraste los 8 revelaron várices. En seis se prac- 
T A B L A No 2

HIPERTENSION PORTAL

OBSTRUCCION PORTAL O ESPLENICA

\begin{tabular}{lcccccccc}
\hline $\begin{array}{l}\text { Número } \\
\text { casos }\end{array}$ & 1 & 2 & 3 & 4 & 5 & 6 & 7 & 8 \\
\hline $\begin{array}{l}\text { RX. Esófago } \\
\text { (Várices) }\end{array}$ & SI & SI & SI & SI & SI & SI & SI & SI \\
$\begin{array}{l}\text { Esplenopor- } \\
\text { tografía }\end{array}$ & SI & NO & SI & SI & SI & SI & NO & SI \\
$\begin{array}{l}\text { Biopsia } \\
\text { hepática }\end{array}$ & $(-)$ & N & N & N & (-) & \pm & (一) & N \\
$\begin{array}{l}\text { Anastomosis } \\
\text { Sobrevida }\end{array}$ & C-M & NO (Tromb.) & C-M & NO & NO & C-M & NO & NO \\
\hline
\end{tabular}

ticó esplenoportografia, la cual demostró en todos ellos obstrucción de la Vena Porta con gran número de colaterales y várices gastroesofágicos. Siete presentaron hematemesis y melena, por lo cual fueron hospitalizados (tabla No 2).

El caso 2 presentó un sindrome de hiperesplenia con plaquetopenia y leucopenia importante. Se consideró que el descenso marcado de las plaquetas era un factor desencadenante de las hemorragias digestivas por lo que se decidió realizar una esplenectomía con el fin de lograr una normalización del número de plaquetas y así lograr una disminución de la frecuencia de hemorragias. Después de la esplenectomía se observó una mejoría por algunos meses. Un año después volvió a consultar por hemorragias repetidas y en esta ocasión se decidió intentar una anastomosis mesentérico cava. En esta 2 a intervención quirúrgica el cirujano describe trombosis de la esplénica, porta y mesentérica, sin encontrar ninguna posibilidad de anastomosis.
Se practicó biopsia hepática en 5 casos: todos mostraron normalidad histológica, excepto el caso 6 , que presentaba discreta fibrosis portal.

Cuatro pacientes fueron operados, en dos (casos 3-6) se realizó una anastomosis cavo-mesentérica con éxito. Uno de ellos en el post-operatorio presentó signos de encefalopatía portal que se prolongó durante dos meses. El tercer caso operado fue el (caso 2) descrito anteriormente en el que no se encontró posibilidad de anastomosis. El cuarto paciente (caso 1) además de hipertensión portal presentaba malformaciones múltiples y un gran retardo pondo estatural. Las hemorragias digestivas en este niño comenzaron a los 2 años 4 meses y en el último año se repetían cada semana, por lo que se decidió intentar una anastomosis. En cse momento de la evolución de su cnfermedad el paciente tenía 4 años de edad y pesaba $10.500 \mathrm{~kg}$. Durante la intervención quirúrgica se comprobó que los calibres de los vasos venosos no eran adecuados para una buena anas.

T A B L, A No 3

HIPERTENSION PORTAL O ESPLENICA

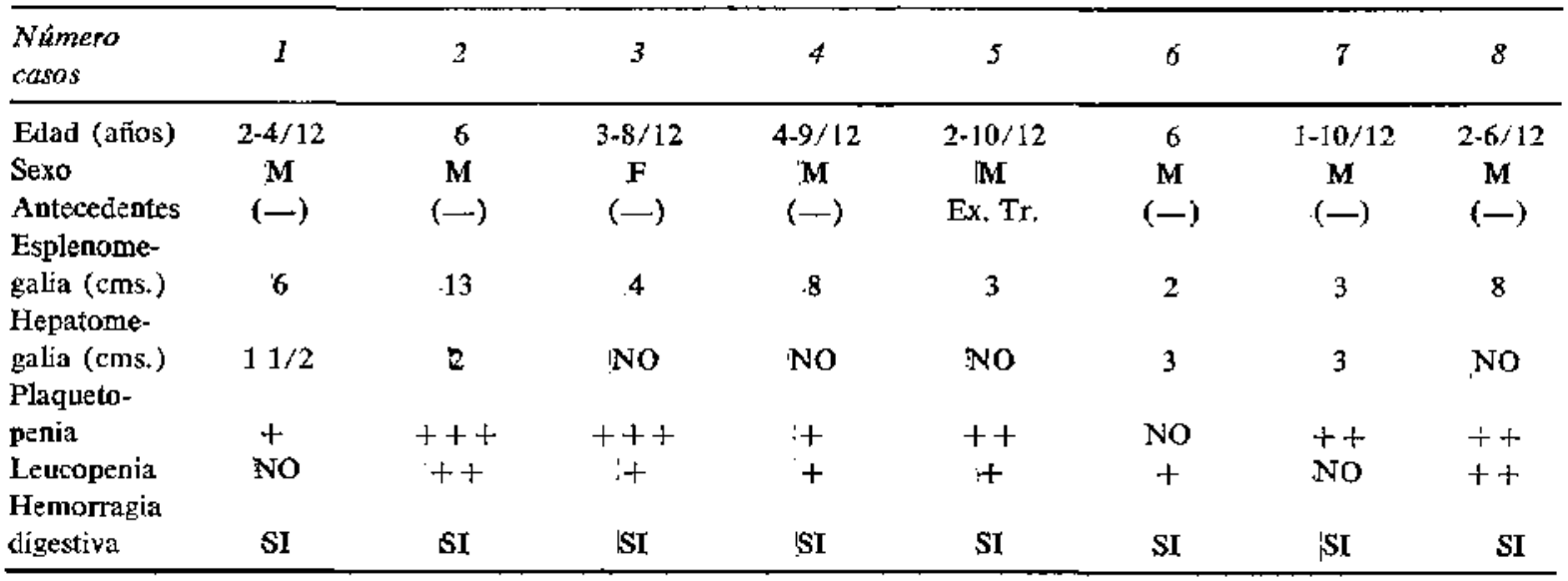


tomosis. Se realizó una anastomosis cavo mesentérica, pero el paciente siguió con hemorragias faIlecicndo 2 horas después de la operación.

En tres de los 12 casos presentados se diagnosticó una Fibrosis hepática congénita.

En la tabla No 3, aparecen las manifestaciones clínicas. Todos eran mayores de 5 años; en dos se encontraron antecedentes de ingestión de agua contaminada con arsénico (ambos enviados de Antofagasta donde el agua potable de un sector de la población estaba contaminada con arsénico). Estos dos pacientes presentaban Iesiones dérmicas de arsenicismo crónico. Se comprobó aumento del bazo en los tres, pero de hepatomegalia sólo en uno. Sólo un paciente presentó hemorragias digestivas a repetición. En dos se comprobó la presencia de várices esofágicos en una radiografía de esófago y en el tercer paciente en la esplenoportografía. En dos casos la esplenoportografía mostró flujo libre en el trayecto esplenoportal, pero dificultad en el llene intrahepático.

No se comprobó alteración de la función hepática. La biopsia hepática quirúrgica practicada en 2 pacientes mostr 6 abundante fibrosis en gruesas bandas limitada a los espacios porta con conductillos biliares dilatados, sin pérdida de la estructura hepática. (Tabla No 4). En el caso 3, se practicó biopsia por punción observándose un espacio porta con abundante tejido fibroso no invasor.

T A B L A No 4

HIPERTENSION PORTAL

FIBROSIS HEPATICA CONGENITA

\begin{tabular}{|c|c|c|c|}
\hline Número casos & 1 & 2 & 3 \\
\hline $\begin{array}{l}\text { Rx. Esófago } \\
\text { (Várices) }\end{array}$ & SI & SI & $\mathbf{N}$ \\
\hline Bateria hepática & $\mathrm{N}$ & $\mathbf{N}$ & $\mathbf{N}$ \\
\hline $\begin{array}{l}\text { Esplenoporto- } \\
\text { grafia }\end{array}$ & SI (alt.) & No & SI (ait.) \\
\hline $\begin{array}{l}\text { Presión } \\
\text { impactada }\end{array}$ & No & $\uparrow$ & $\mathbf{N}$ \\
\hline Biopsia & & & \\
\hline $\begin{array}{l}\text { bepática } \\
\text { Anastomosis }\end{array}$ & $\begin{array}{c}+++ \\
\text { Operación }\end{array}$ & $\begin{array}{l} \pm++ \\
\text { INO }\end{array}$ & $\begin{array}{l}+ \\
\text { No }\end{array}$ \\
\hline Sobrevida & SI & Sf & SI \\
\hline
\end{tabular}

La presión venosa impactada resultó normal en uno y discretamente elevada en el otro. Los tres se encuentran vivos y en buenas condiciones, excepto el caso 1 , que fue sometido a repetidas intervenciones sin que fuera posible realizar una anastomosis. En la última intervención se ligaron los colaterales del lecho portal con lo cual se logró una franca disminución de las hemorragias.
Sólo un caso de cirrosis hepática con $\mathbf{H}$, P. fue controlado durante Ios 6 años en que se estudiaron estos enfermos. Era una niña de 11 años que en la fase terminal de una hepatitis crónica activa presentó una cirrosis postnecrótica con Hipertensión Portal.

Comentario. Recientemente se ha sugerido la siguiente agrupación de las diversas causas de Hipcrtensión Portal (2).

A. Presinusoidal:

Extra hepática: $\left\{\begin{array}{l}\text { Obstrucción de la esplénica } \\ 0 \text { porta. }\end{array}\right.$

Intra hepática: $\left\{\begin{array}{l}\text { Fibrosis hepática congénita } \\ \text { Enfetmedad de Wilson } \\ \text { Esquistosomiasis } \\ \text { Enfermedades infiltrativas }\end{array}\right.$

B. Post-sinusoidal:

Intra hepática: $\left\{\begin{array}{l}\text { Cirrosis hepática } \\ \text { Enfcrmedad venooclusiva }\end{array}\right.$

Extra hepática: Obstrucción vena suprahepática

Las causas más frecuentes de Hipertensión Portal en los casos estudiados fueron la obstrucción de la Vena Porta y en segundo lugar la Fibrosis hepática congénita. Se controló sólo un caso de cirrosis hepática con Hipertensión Portal en una niña de $1 \mathfrak{l}$ años con hepatitis crónica activa.

Se han sugerido numerosas causas en la etiología de la trombosis de la Vena Porta, pero excepcionalmente se encuentra el antecedente de una de ellas en la anamnesis de estos pacientes.

En uno de los 8 casos aquí presentados se confirmó el antecedente en el período de Recién Nacido de una exanguíneo transfusión sin infección umbilical posterior.

Walker (3) en un control a largo plazo de 3.000 Recién Nacidos sometidos a exanguíneo transfusión encontrố sólo 1 caso de trombosis portal.

Thompson (4) en 86 casos con infección umbilical en período de Recién Nacido no encontró ningún caso de trombosis portal.

El diagnóstico de Hipertensión portal por trombosis de la porta debe plantearse en aquellos niños que presentan hemorragjas digestivas (hematemesis y melena.), esplenomegalia y un desarrollo pondo estatural normal. En los pacicntes mayores de 5 años que presentan además de lo descrito una hepatomegalia, debe considerarse la posibilidad de una fibrosis hepática congénita. En esta última afección, el diagnóstico difercncial se 
fundamentará en la esplenoportografía que muestra un flujo esplenoportal libre con gruesas colaterales y várices gastroesofágicos y dificultad de llene intrahepático, y en la biopsia hepática quirúrgica donde $\mathrm{se}$ evidencian las alteraciones bistológicas típicas de esta enfermedad. La biopsia hepática por punción es insuficiente para confirmar el diagnóstico. Cuando la Hipertensión Portal es provocads por cirrosis hepática se observa con frecuenica manifestaciones clínicas $y / 0$ de laboratorio de daño hepático, además de deficiente desarrollo pondoestatural. En estos casos la biopsia hepática confirma el diagnóstico.

Es interesante hacer notar que en 5 de nuestros enfermos los síntomas de hipertensión portal aparccieron antes de los 4 años de edad. Este hecho guarda estrecha rélación con el pronóstico ya que sabemos que en niños menores de 6 años las posibilidades de corrección son muy limitadas y casi nula bajo Ios 4 años. Cuando el sindrome de hiperesplenia mantiene una plaquetopenia importante no se debe realizar esplenectomía como medida paliativa, porque con frecuencia se produce trombosis de la esplénica comprometicndo además lo que queda libre de la porta y la mescntérica impidiendo así toda posibilidiad de anastomosis en un periodo posterior (5).

En los niños menores de 4 años con hemorragias a repetición se debe intentar otros tratamicntos paliativos permitieudo así unia mayor edad para realizar una anastomosis con mejores posibilidades. De estos procedimientos quizás el más eficiente sería la ligadura de las várices gastroesofágicas y colaterales porta.

En algunos casos se observa que al pasar los años las frecuencias de las hemorragias digestivas disminuyen paulatinamente por recanalización de la zona trombosada.

A diferencia de los pacientes con cirrosis hepática e hipertensión portal, los enfermos con trombosis de la porta o con Fibrosis hepática congénita tienen muy buen pronóstioo cuando se soluciona el problema vascular, ya que la función hepática es normal.

Es interesante el hecho de que dos pacientes con Fibrosis Hepútica congénita tuvieran síntomas manifiestos de arsenicisno crónico. De la experiencia obtenida en los numerosos casos de intoxicaciones crónicas con arsénico observada en algunas poblaciones de Antofagasta, sabemos que estos producen lesiones vasculares importantes. Así se han descrito niños con infarto al miocardio, lesiones vasculares de extremidades y otras (6). Si consideramos que en las Fibrosis hepáticas congénitas se describe además una hipoplasia de la ramificación porta (7), quedaría por investivar el posible papel que pucde tener el arsénico en esta patología.

\section{RESUMEN}

Se revisaron las caracteristicas clinicas y de laboratorio de 12 pacientes con Hipertensión Portal estudiados durante el período 1968-1974.

Se encontraron las siguientes causas de $\mathrm{Hi}$ pertensión Portal:

8 Casos con Obstrucción de la Porta.

3 Casos con Fibrosis Hepática Congénita.

1 Caso de Hepatitis Crónica Activa y Cirrosis Hepatica.

$S_{\mathcal{C}}$ analizan algunos aspectos del Diagnóstico diferencial, utitidad de los exámenes e indicaciones de tratamiento quirúrgico.

\section{SUMMARY}

Clinical and laboratory data of twelve patients with Portal Hypentension who were admitted to the Hospital "Luis Calvo Mackonna" between the years 1968-1974 was analised.

Portal hypertension in these patients was caused by the following pathology:

Portal trombosis in 8 patients.

Hepatic Fibrosis in 3 patients.

Hepatic Cirrhosis in 1 patient.

We comment upon clinical and laboratory fidings which are useful in tre differential diagnosis and on the surgical management of Pediatric patients.

\section{REFERENCIAS}

I.-Clatwarty H, W, and de Lorimier, A, A. Am. J. Surgery 107: 447, 1964.

2.- Sherlock Sheila: "Hepatic Circulatory Changes in munn". Chapter 13. Pág. 165. "Currents Concepts of Clinical Gastroentcrology" J. and A. Churchill Ltda., London 1965.

3.-Walker W. Mencionado por Wilson, K. W. Robinson D. C. y Hacking P. $M$. como comunicación personal en Brit. J. Surg. 56: 13, 1969.

4.-Thompson, E. N. and Sherlock, S. Quat J. Med., 33: $465,1964$.

5.- Silverman A. Roy C. and Cozzetho, F. Pediatric Clinical Gastroenterology. Ed. Mosby Company Saint Louis, 1971, pág. 424.

6.-Roscmbery, H. "Sistemic Arterial disease with Myocardial infarction" Report of 2 infants. Circulation 47: 270-275, 1973.

7.-Zucuras J., del Pozo H. y Brinck P. Revista Chilena de Pediatría, 42: 429, 1971. 